\title{
Prevalência de anemia ferropriva em gestantes brasileiras: uma revisão dos últimos 40 anos $^{1}$
}

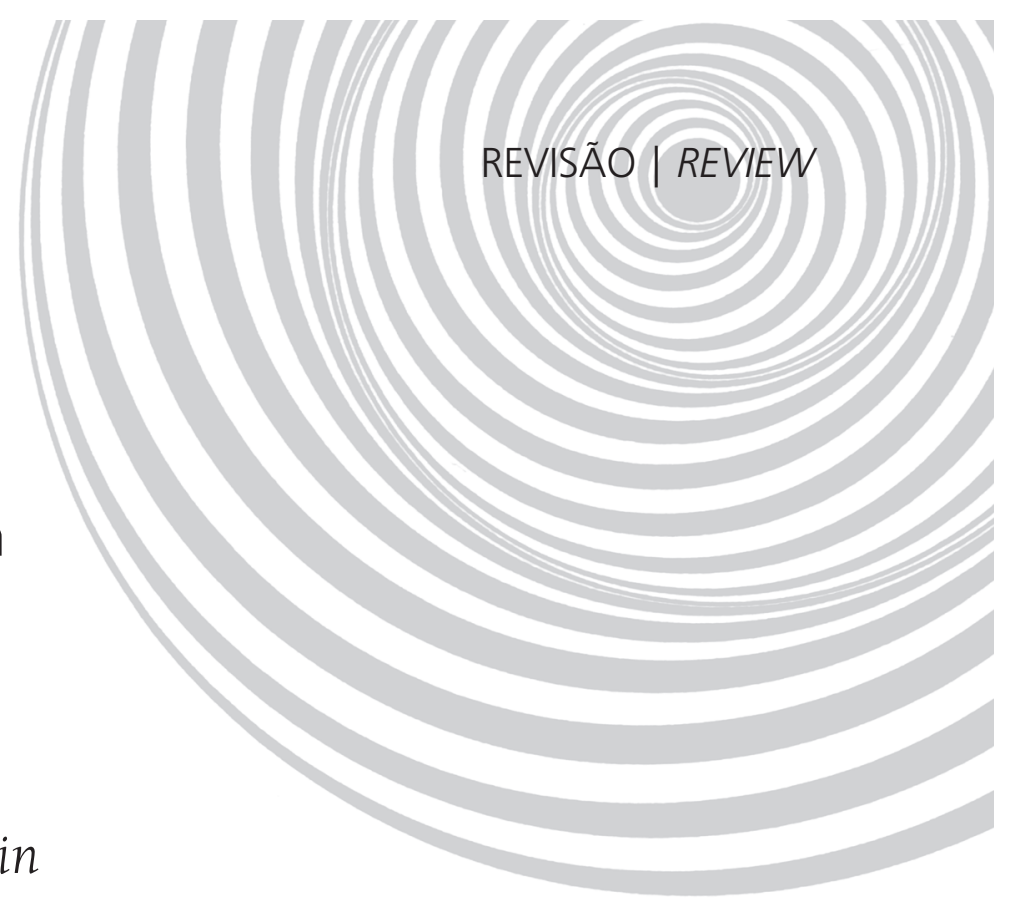

\author{
Prevalence of iron-deficiency anemia in \\ Brazilian pregnant women: a review \\ of the last 40 years
}

Mariana Helcias CÔRTES²

Ivana Aragão Lira VASCONCELOS²

Denise Costa COITINHO

RES U M O

A anemia ferropriva é conhecida como uma das principais deficiências nutricionais em todo o mundo e sua ocorrência pode ser observada em diversas populações. Alguns grupos populacionais ainda apresentam altas prevalências de anemia ferropriva, comprometendo diversas funções do organismo. Dentre esses grupos de risco, podem ser citadas as gestantes, que merecem especial atenção devido à sua vulnerabilidade à carência e ao aumento significativo de suas necessidades, que não são acompanhados por aumento suficiente no consumo ou na absorção de ferro. Este artigo analisa dados de artigos publicados nos últimos 40 anos nas línguas inglesa, espanhola ou portuguesa nos bancos de dados Lilacs e Medline sobre prevalência de anemia ferropriva na gestação. Em todas as pesquisas verificadas, a prevalência de anemia ferropriva na gestação apresenta valores elevados, o que caracteriza essa situação como um problema de saúde pública para o Brasil, mesmo com as políticas nacionais atuais de combate à deficiência.

Termos de indexação: Anemia ferropriva. Brasil. Gestantes. Prevalência.

A B S T R A C T

Iron-deficiency anemia is known as one of the main nutritional deficiencies around the world and its occurrence can be observed in many populations. Some groups still present a high prevalence of iron-deficiency anemia,

1 Artigo elaborado a partir da dissertação de M.H. CÔRTES, intitulada "Impacto da fortificação das farinhas de trigo e de milho com ferro nos níveis de hemoglobina das gestantes atendidas pelo pré-natal do Hospital Universitário de Brasília/DF". Universidade de Brasília; 2006.

2 Universidade de Brasília, Faculdade de Ciências da Saúde, Programa de Pós-Graduação em Nutrição Humana, Departamento de Nutrição. Campus Universitário Darcy Ribeiro, Asa Norte, 70910-900, Brasília, DF, Brasil. Correspondência para/Correspondence to: M.H. CÔRTES.E-mails:<marianahelcias@gmail.com> ; <mariana.helcias@mds.gov.br>.

3 Programa Mundial de Alimentos das Nações Unidas, Coordenação da Força-Tarefa Interagencial para o Fim da Fome e Desnutrição na Infância. Roma, Itália. 
410 | M.H. CÔRTES et al.

compromising many body functions. Pregnant women are among the groups at risk given their vulnerability to deficiencies and the significant increase in their requirements, which are not accompanied by a sufficiently higher iron intake or absorption. This paper analyzes data from articles published in the last 40 years in English, Spanish or Portuguese found in the Lilacs and Medline databases about the prevalence of iron-deficiency anemia during pregnancy. Every paper found reports a high prevalence of anemia in pregnant women, characterizing this situation as a public health problem in Brazil, despite current Brazilian policies to fight iron deficiency.

Indexing terms: Iron-deficiency anemia. Brazil. Pregnant woman. Prevalence.

\section{N T R O D U Ç Ã O}

Dentre as deficiências nutricionais mais presentes em todo o mundo, a anemia por deficiência de ferro, ou anemia ferropriva, é reconhecida como uma das mais relevantes, especialmente pelo fato de que qualquer grupo etário é vulnerável a essa deficiência ${ }^{1-3}$. A anemia é definida pela Organização Mundial da Saúde (OMS) - como aumento ou diminuição do tamanho das hemácias acompanhada da redução ou não da concentração de hemoglobina ${ }^{4}$. A anemia nutricional é aquela cuja etiologia está relacionada à carência de um ou mais nutrientes, sendo a deficiência no consumo ou na absorção de ferro suas causas mais comuns ${ }^{4}$.

No Brasil, a anemia tem sido encontrada em vários estados do território nacional e se diferencia de outras condições carenciais por não se limitar a acometer apenas as populações de mais baixa renda ou apenas os desnutridos 5 . Contudo, a anemia ferropriva compromete, principalmente, alguns grupos mais sensíveis à escassez de ferro devido ao crescimento rápido ou ao aumento de demanda: crianças entre seis meses e cinco anos de idade, adolescentes do sexo feminino, mulheres em idade fértil, 2,6 gestantes e nutrizes ${ }^{1}$.

No caso particular da gestação, além da expansão do volume sangüíneo ${ }^{6}$, também ocorre aumento de demanda pelas necessidades do feto ${ }^{3}$. Além disso, é pequeno o número de mulheres que iniciam a gestação com os estoques de ferro adequados ${ }^{7,8}$. A falta de ferro no organismo aumenta as mortalidades infantil e materna e prejudica a produtividade ${ }^{9,4}$. Ainda, quando a hemoglobina encontra-se significativamente abaixo dos níveis adequados, a gestante pode sofrer de insu- ficiência cardíaca de alto débito, com risco de morte para ela e para o bebêe, ${ }^{8,10}$.

Dessa forma, com base nos dados existentes que demonstram tanto a elevada prevalência dessa deficiência no Brasil ${ }^{11-13}$ quanto as conseqüências dessa carência ${ }^{1,4,8-10}$, objetivou-se realizar um levantamento da prevalência de anemia no Brasil, com especial enfoque nas gestantes adultas.

\section{MÉTOD OS}

Foi realizada uma busca por meio dos bancos de dados Lilacs e Medline e por artigos publicados nos últimos 40 anos. As palavras-chave incluídas na pesquisa foram: anemia/deficiência de ferro e gestantes; Brasil e prevalência de anemia; hemoglobina/ferritina e gestantes. A pesquisa buscou artigos principalmente em português, por se tratarem de estudos brasileiros, além de artigos em inglês e espanhol.

Além da pesquisa feita pelo banco de dados, outros estudos de interesse foram solicitados aos serviços de bibliotecas especializados. A revisão foi, basicamente, composta por estudos transversais. Esses estudos foram separados por décadas para facilitar a compreensão e para compor uma linha temporal. Os estudos com adolescentes foram excluídos.

Para os estudos de prevalência de anemia procuraram-se dados sobre os autores, o ano e a cidade onde foi realizado; tipo de estudo conduzido; tamanho, seleção e caracterização da amostra como sexo, idade, número da amostra, região (rural ou urbana); método pelo qual a hemoglobina foi dosada no estudo, ponto de corte considerado e a prevalência encontrada. 


\section{RESULTADOS}

\section{Estudos década de 1970 (Tabela 1)}

Cook et al. ${ }^{14}$, em estudo realizado em 1971, investigaram a anemia nutricional em 889 gestantes de terceiro trimestre em sete países da América Latina, incluindo 83 gestantes brasileiras. A deficiência de ferro (saturação de transferrina $<15,0 \%$ ) foi encontrada em $48,0 \%$ do total de gestantes estudadas, e a anemia em $38,5 \%$. O valor da hemoglobina variou de 10,2 a 12,2g/dL.

Almeida et al. ${ }^{15}$ investigaram 701 gestantes matriculadas no Serviço de Pré-natal da Faculdade de Saúde Pública da Universidade de São Paulo, submetidas a três ou quatro dosagens em vários meses de gestação (total de 2099 dosagens) no período de 1947 a 1969. Neste estudo é possível observar, para cada mês gestacional investigado, o número de dosagens realizadas e a respectiva prevalência de anemia. Para o segundo mês gestacional esta prevalência se encontra em $10,6 \%$, elevando-se gradativamente com o passar do período gestacional (para 15,2\% no terceiro mês, depois para 15,9\% no quarto, $20,1 \%$ para o quinto e $20,9 \%$ para o sexto), atingindo seu ponto máximo em torno dos meses sete $(20,1 \%)$ e oito $(22,1 \%)$ e voltando a cair para $20,5 \%$ no último mês de gestação.

O estudo de Szarfarc ${ }^{16}$, pesquisou 263 parturientes a termo, atendidas na Casa Maternal e de Assistência à Infância da Legião Brasileira de Assistência, em São Paulo. Observou-se uma alta prevalência de anemia (52,3\%), sendo que o pon- to de corte para anemia foi hemoglobina $<12 \mathrm{~g} / \mathrm{dL}$ e hematócrito $<35,0 \%$. Dentre as anêmicas, $16,3 \%$ tinham anemia severa $(\mathrm{Hb} \leq 10 \mathrm{~g} / \mathrm{dL}$ e/ou hemotócrito $\leq 30,0 \%$ ) e 36,0\% anemia moderada $(10 \mathrm{~g} / \mathrm{dL}<\mathrm{Hb}<12 \mathrm{~g} / \mathrm{dL})$. Trinta e nove por cento das parturientes apresentaram deficiência de ferro,

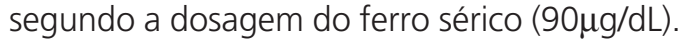

Em 1975, Vaz Pinto et al. ${ }^{17}$ encontraram, entre 407 gestantes atendidas pelo Serviço de Obstetrícia da Unidade Integrada de Saúde em Sobradinho, Distrito Federal (DF), prevalência de anemia em $28,0 \%$ da amostra. Entre as gestantes do primeiro trimestre havia $22,0 \%$ de anêmicas, $29,0 \%$ entre as de segundo trimestre e $34,0 \%$ nas de terceiro trimestre. Os autores utilizaram o ponto de corte para anemia de $12 \mathrm{~g} / \mathrm{dL}$. No grupo com anemia, a deficiência de ferro foi representada por $73,7 \%$.

Roncada \& Szarfarc ${ }^{18}$ averiguaram duas zonas urbanas do Vale do Ribeira: Apiaí e Ribeira (no estado de São Paulo). Observaram maior prevalência de anemia em gestantes de Ribeira $(57,1 \%)$ em relação às de Apiaí $(23,8 \%)$. A prevalência não foi subdividida por trimestre de gestação.

\section{Estudos década de 1980 (Tabela 2)}

Salzano et al. ${ }^{19}$ investigaram a prevalência da anemia no ciclo gestacional em 472 gestantes de Pernambuco e em 217 da Paraíba. A prevalência de anemia em quatro áreas urbanas de Pernambuco foi de $33,7 \%$ e em oito áreas rurais

Tabela 1. Estudos de prevalência de anemia em gestantes no Brasil na década de 1970. Brasília (DF), 2006.

\begin{tabular}{|c|c|c|c|c|}
\hline Autor & Local & Amostra & Método de dosagem da hemoglobina & Pontos de corte $\mathrm{g} / \mathrm{dL}$ \\
\hline Cook et al. ${ }^{14}$ & $\begin{array}{l}\text { Sete Países da } \\
\text { América Latina }\end{array}$ & 889 gestantes & Cianometahemoglobina & 11,0 \\
\hline Almeida et al. ${ }^{15}$ & São Paulo (SP) & 701 gestantes & Oxihemoglobina & 13,2 a 16,7 \\
\hline Szarfarc ${ }^{16}$ & São Paulo (SP) & 263 parturientes & Cianometahemoglobina & $\mathrm{Hb}<12,0$ e $\mathrm{Hc}<35 \%$ \\
\hline Vaz Pinto et al. ${ }^{17}$ & $\begin{array}{l}\text { Sobradinho, } \\
\text { Brasília (DF) }\end{array}$ & 407 gestantes & Cianometahemoglobina & 12,0 \\
\hline Roncada \& Szarfarc ${ }^{18}$ & Vale do Ribeira (SP) & 60 gestantes & Não mencionado & 11,0 \\
\hline
\end{tabular}


412 | M.H. CÔRTES et al.

Tabela 2. Estudos de prevalência de anemia em gestantes no Brasil na década de 1980. Brasília (DF), 2006.

\begin{tabular}{|c|c|c|c|c|}
\hline Autor & Local & Amostra & Método de dosagem da hemoglobina & Pontos de corte $\mathrm{g} / \mathrm{dL}$ \\
\hline Salzano et al. ${ }^{19}$ & Pernambuco & $\begin{array}{l}472 \text { gestantes de } \\
\text { quatro áreas urbanas }\end{array}$ & Cianometahemoglobina & 11,0 \\
\hline Salzano et al. ${ }^{18}$ & Paraíba & $\begin{array}{l}217 \text { gestantes de sete } \\
\text { áreas rurais }\end{array}$ & Cianometahemoglobina & 11,0 \\
\hline Szarfarc et al. ${ }^{20}$ & São Paulo (SP) & $\begin{array}{l}228 \text { Gestantes } \\
>36 \text { semanas }\end{array}$ & Não mencionado & 11,0 \\
\hline Romani et al. ${ }^{21}$ & Recife (PE) & $\begin{array}{l}461 \text { gestantes de um } \\
\text { Centro de saúde }\end{array}$ & Cianometahemoglobina & 11,0 \\
\hline Romani et al. ${ }^{21}$ & Recife (PE) & $\begin{array}{l}249 \text { gestantes de um } \\
\text { Centro de saúde }\end{array}$ & Cianometahemoglobina & 11,0 \\
\hline Szarfarc ${ }^{22}$ & Estado de São Paulo & $\begin{array}{l}4539 \text { gestantes em } 15 \\
\text { Centros de saúde do estado }\end{array}$ & Cianometahemoglobina & 11,0 \\
\hline
\end{tabular}

da Paraíba foi de 36,9\%. A prevalência de anemia foi semelhante em ambos os estados, destacando-se apenas dois municípios de Pernambuco que apresentaram uma prevalência acima da média $(80,0 \%)$.

Szarfarc et al. ${ }^{20}$ avaliaram a influência do número de consultas do atendimento pré-natal na deficiência de ferro entre gestantes de 36 semanas ou mais, em comparação ao atendimento pré-natal satisfatório, todas atendidas no parto pela maternidade da Fundação Amparo Maternal e no Serviço de Pré-natal do Centro de Saúde-Escola Geraldo de Paula Souza, da Faculdade de Saúde Pública, Universidade de São Paulo. As participantes foram distribuídas em três grupos: padrão (com atendimento pré-natal satisfatório); com algum atendimento pré-natal (CPN); e sem pré-natal (SPN). Os grupos CPN e SPN tiveram cerca de 3 e 6,5 vezes mais anêmicas que o grupo padrão, respectivamente. As gestantes do grupo padrão apresentaram níveis de concentração de hemoglobina significativamente maiores que as do grupo CPN e ainda maiores do que as do grupo SPN. Ainda, houve diferenças estatisticamente significantes quando as médias de hemoglobina das três populações foram comparadas duas a duas. Os autores aconselham a necessidade do aumento da cobertura e da uniformização na qualidade dos serviços de pré-natal.
A pesquisa de Romani et al. ${ }^{21}$ estimou a prevalência de anemia em 710 gestantes de duas grandes unidades de saúde do Recife (PE): o Centro de Saúde Lessa de Andrade (CSLA) e o Posto de Assistência Médica de Areias (PAMA), com prevalências de anemia de $39,8 \%$ e $8,9 \%$, respectivamente. Além de ter tido a maior prevalência, o CSLA obteve ocorrência de anemia nas formas mais severas. O período gestacional apresentou variação significativa da hemoglobina entre o primeiro e o segundo trimestres e entre o primeiro e o terceiro trimestres, sendo maior no primeiro trimestre. No CSLA foi observado $23,9 \%$ de anemia no $1^{\circ}$ trimestre; $43,9 \%$ no $2^{\circ}$ e $41,6 \%$ no $3^{\circ}$ trimestres. Já no PAMA as prevalências encontradas, respectivamente, foram de $6,4 \%$ para o $1^{\circ}$ trimestre; $9,2 \% \%$ para $2^{\circ}$ e $10,9 \%$ para o $3^{\circ}$ trimestres.

Um estudo feito por Szarfarc ${ }^{22}$ em 15 unidades de saúde de São Paulo (38,5\% do total existente na época), com 4539 gestantes, constatou prevalência de anemia de $35,1 \%$. As prevalências entre os centros de saúde tiveram grande variação - de 7,8\% a 65,2\%. Separando as gestantes por idade gestacional, o trabalho encontrou tendência de manutenção dos valores de hemoglobina no início da gestação, seguida de diminuição e posterior aumento leve no final do processo. 


\section{Estudos década de 1990 (Tabela 3)}

No período de abril a outubro de 1988 , 363 gestantes de primeira consulta e que não usavam suplementos de ferro, ácido fólico ou vitamina B12 foram estudadas por Guerra et al. ${ }^{23}$. Eram gestantes de oito centros de saúde do Butantan, em São Paulo, e apresentaram uma prevalência geral de anemia de $12,4 \%$, sendo $3,6 \%$ para o primeiro, $20,9 \%$ para o segundo e do $32,1 \%$ para o terceiro trimestres. A prevalência de anemia no primeiro trimestre foi significativamente menor que as prevalências do segundo e terceiro trimestres. As gestantes que tiveram mais de três partos apresentaram prevalência de anemia significativamente maior do que aquelas com até três partos. Aquelas com renda de até 0,5 salário-mínimo per capita apresentaram prevalência maior da deficiência.

Sinisterra-Rodriguez ${ }^{24}$ estudou a anemia em 691 gestantes atendidas no parto (terceiro trimestre) do Hospital Maternidade Leonor Mendes de Barros, em São Paulo. Foram identificadas $29,2 \%$ de gestantes anêmicas. Não se observou associação entre a freqüência da anemia e o atendimento pré-natal nem entre anemia e desnutrição materna, porém houve associação entre o baixo peso ao nascer e a anemia. Além disso, a hemoglobina de mulheres que usaram suplemento de ferro foi estatisticamente maior em relação àquelas que não o utilizaram.
No início da década de 1990, Arruda ${ }^{13}$ estudou 710 gestantes atendidas em primeira consulta no ambulatório de pré-natal e 386 gestantes admitidas em trabalho de parto na maternidade do Instituto Materno-Infantil de Pernambuco (IMIP). No primeiro grupo de gestantes, a média de hemoglobina foi de $11,4 \mathrm{~g} / \mathrm{dL}$ (7,6-16,3g/dL), sendo que havia $30,3 \%$ de anêmicas no grupo. Nas gestantes estudadas no pré-natal, verificou-se associação estatisticamente positiva entre anemia e trimestre gestacional, presença de parasitas intestinais espoliadores de ferro, nível educacional e estado nutricional; associações mantidas com o controle de variáveis. Não foi encontrada associação entre anemia e risco gestacional, número de filhos, intervalo interpartal, hábito de fumar e idade. Observou-se que 17,2\% das gestantes de primeiro trimestre eram anêmicas, enquanto essa prevalência subiu para $29,1 \%$ e $44,3 \%$ no segundo e no terceiro trimestres, respectivamente. Com relação ao nível de escolaridade, foi verificada associação inversa entre anemia e escolaridade. Foi evidenciada maior prevalência de anemia entre as gestantes de baixo peso, enquanto $29 \%$ das gestantes com peso normal apresentaram baixos níveis de hemoglobina.

No grupo da maternidade, a hemoglobina materna teve média de $11,3 \mathrm{~g} / \mathrm{dL}(5,0-6,9 \mathrm{~g} / \mathrm{dL})$ e a prevalência de anemia foi de 38,3\%. Observou-se tendência sem significância estatística de maior

Tabela 3. Estudos de prevalência de anemia em gestantes no Brasil na década de 1990. Brasília (DF), 2006.

\begin{tabular}{|c|c|c|c|c|}
\hline Autor & Local & Amostra & Método de dosagem da hemoglobina & Pontos de corte $\mathrm{g} / \mathrm{dL}$ \\
\hline Arruda $^{13}$ & Recife (PE) & 710 gestantes & Cianometahemoglobina & 11,0 \\
\hline Arruda $^{13}$ & Recife (PE) & 386 gestantes & Cianometahemoglobina & 11,0 \\
\hline Guerra et al. ${ }^{23}$ & São Paulo (SP) & 363 gestantes & Cianometahemoglobina & 11,6 \\
\hline Sinisterra Rodriguez ${ }^{24}$ & São Paulo (SP) & 691 gestantes & Não mencionado & 11,0 \\
\hline Nacul et al. ${ }^{25}$ & Recife (PE) & 1508 gestantes & Espectrofotometria & 11,0 \\
\hline Cardoso et al. ${ }^{26}$ & $\begin{array}{l}\text { Candeias do Jamari, } \\
\text { Município de Porto } \\
\text { Velho (RO) }\end{array}$ & $\begin{array}{l}1068 \text { indivíduos de } \\
\text { todas as idades }\end{array}$ & Cianometahemoglobina & 11,0 \\
\hline Arruda ${ }^{27}$ & Recife (PE) & 1007 gestantes & Cianometahemoglobina & 11,0 \\
\hline Rondó \& Tomkins 28 & Campinas (SP) & 712 parturientes & Cianometahemoglobina & 11,0 \\
\hline
\end{tabular}


risco de anemia em bebês nascidos de mães anêmicas. Também nesse grupo foi evidenciada associação entre escolaridade e anemia, sendo a prevalência de anêmicas de 55\% entre as mulheres sem instrução e de $26,2 \%$ entre as que tinham de cinco a sete anos de estudo. Não foi encontrada associação estatística entre anemia e o estado nutricional ou o tempo de duração da gestação ${ }^{13}$.

Nacul et al. ${ }^{25}$, em um estudo populacional, pesquisaram a prevalência de anemia em 1508 gestantes atendidas no ambulatório de pré-natal do IMIP entre 1987 e 1988. Mais de $25,0 \%$ apresentaram hemoglobina inferior a $11 \mathrm{~g} / \mathrm{dL}$. Foi encontrada anemia três ou quatro vezes maior entre gestantes de segundo e terceiro trimestres em relação ao primeiro. Os resultados também evidenciaram que as maiores ocorrências de anemia foram encontradas em primigestas e gestantes com três ou mais gestações.

Cardoso et al. ${ }^{26}$ estudaram a prevalência de anemia em município endêmico para malária. Foram analisados 1068 sujeitos de todas as idades, independentemente da condição de saúde e do estado fisiológico. Das 17 gestantes amostradas, sete $(41,2 \%)$ eram anêmicas e $28,0 \%$ da amostra apresentaram essa mesma condição.

Arruda realizou um estudo descritivo seccional em 1997, na maternidade do IMIP. Foram avaliadas 1007 gestantes atendidas em trabalho de parto. Do total de mulheres avaliadas, $30,9 \%$ apresentaram anemia, sendo que em $2,6 \%$ delas a hemoglobina se encontrava abaixo de $9 \mathrm{~g} / \mathrm{dL}$. A média de hemoglobina encontrada foi de 10,1 g/dL (desvio-padrão - DP=0,9). Nesse estudo, houve associação estatística entre escolaridade e anemia, sendo o Odds Ratio (OR) para a associação de 2,09 (IC 95\%: 1,13-3,88) 27.
A pesquisa de Rondó \& Tomkins ${ }^{28}$, de 1999 comparou o status de ferro de 356 parturientes com conceptos que tiveram retardo no crescimento intra-uterino (IUGR) com 356 parturientes com conceptos de peso adequado para idade gestacional (ADA). O estudo foi realizado em quatro hospitais de Campinas (estado de São Paulo), correspondendo a 95,0\% dos partos entre 1991 e 1992. Quarenta e sete por cento da amostra de mães eram anêmicas. A média do nível de hemoglobina foi maior em mães com filhos IUGR $(11,2$, $D P=1,73 \mathrm{~g} / \mathrm{dL}$ ) que em mães com filhos $A D A$ $(10,96, D P=1,7 \mathrm{~g} / \mathrm{dL})$ e a anemia foi mais prevalente em mães com filhos $\operatorname{ADA}(p=0,004)$. Altos níveis de ferritina foram estatisticamente mais comuns em mães com filhos IUGR $(p<0,001)$. Houve correlação entre a hemoglobina do sangue fetal e materno para ambos os grupos.

\section{Anos 2000 (Tabela 4)}

Os poucos estudos realizados no início desta década não tratam a prevalência de anemia em gestantes como o principal objetivo do artigo científico. Souza et al. ${ }^{29}$ focalizaram a estimativa da freqüência de enteroparositoses de gestantes em pré-natal de baixo risco e a associação com à anemia. Encontraram, entre 316 gestantes com até 20 semanas de gestação atendidas no pré-natal do IMIP, 55,4\% de prevalência de anemia, com a média de 10,8g/dL, DP=0,8 para a hemoglobina. Não houve associação entre as enteroparasitoses e a anemia $(p=0,091)$, embora ambas as prevalências tenham sido elevadas.

O estudo de Rocha et al. ${ }^{30}$ investigou 168 gestantes atendidas no pré-natal do único centro de saúde disponível no município de Viçosa (esta-

Tabela 4. Estudos de prevalência de anemia em gestantes no Brasil a partir do ano 2000. Brasília (DF), 2006.

\begin{tabular}{llccc}
\hline Autor & Local & Amostra & Método de dosagem da hemoglobina & Pontos de corte g/dL \\
\hline Souza et al..29 & Recife (PE) & $\begin{array}{c}316 \text { gestantes de } \\
\text { até } 20 \text { semanas }\end{array}$ & Oxihemoglobina & 11,0 \\
Rocha et al. ${ }^{30}$ & Viçosa (MG) & 168 gestantes & $\beta$-hemoglobinômetro portátil & 11,0 \\
\hline
\end{tabular}


do de Minas Gerais) para a baixa renda. Foi encontrada prevalência de $21,4 \%$ de mulheres anêmicas, sendo que foram encontradas prevalências de 5,6\%, 20,3\% e 26,3\% para o primeiro, o segundo e o terceiro trimestres, respectivamente. As variáveis antropométricas maternas apresentaram correlação com o peso ao nascer, porém não houve relação entre o estado nutricional de ferro e peso ao nascer. Os autores concluem que a anemia na gravidez pode ser devida à qualidade de assistência pré-natal relacionada à prevenção e ao tratamento da anemia ferropriva, por meio da suplementação medicamentosa.

\section{I S C U S S Ã O}

No Brasil, a anemia em gestantes tem sido estudada por alguns autores desde a década de 1970, e a maior parte dos estudos data dos anos 1970 a 1990. Os resultados encontrados têm variado segundo: os critérios de seleção da amostragem; a idade gestacional da amostra; o método de dosagem da hemoglobina ou ferritina; o ponto de corte utilizado para diagnosticar a anemia; as condições socioeconômicas das gestantes; o estado de saneamento básico da região estudada; o atendimento pré-natal durante a gravidez. Para a classificação da anemia, geralmente é utilizado o critério definido pela Organização Mundial de Saúde (OMS), ou seja, a gestante seria anêmica se a hemoglobina fosse menor que $11 \mathrm{~g} / \mathrm{dL}$. Praticamente todos os estudos que abordam a anemia em gestantes têm sido do tipo transversal e poucos dos tipos caso-controle ou coorte.

As experiências brasileiras, no decorrer das décadas, apresentam resultados discordantes na prevalência de anemia em gestantes, até mesmo quando são aplicados os mesmos procedimentos metodológicos nas mesmas regiões. Como resultados dessas variações entre os estudos, foram encontradas prevalências de anemia no primeiro trimestre variando de 3,6\%, no estudo de Guerra et al. ${ }^{23}$; a 23,9\%, na pesquisa de Romani et al. ${ }^{21}$. No segundo trimestre os resultados oscilam entre $9,2 \%$ e $43,9 \%$, e, no terceiro trimestre, variam de $10,9 \%$ no mesmo estudo a $52,3 \%{ }^{16}$. Alguns estudos de décadas variadas ${ }^{15,17,21,23,25,30,31}$ encontraram maior prevalência de anemia com o avançar do período gestacional.

Deve-se ressaltar que muitas gestantes iniciam o acompanhamento de pré-natal apenas depois do final do primeiro trimestre gestacional, o que contribui para o maior número de estudos realizados a partir do segundo trimestre. Apesar de a maior prevalência de anemia ser encontrada no terceiro trimestre, o estudo de coorte realizado por Szarfarc et al. ${ }^{31}$ e um estudo de Almeida et al. ${ }^{15}$, que mostrou uma curva de hemoglobina durante a gestação, observaram um leve aumento no nível de hemoglobina a partir do sétimo mês até o final da gestação.

Grande parte dos estudos foi realizada no estado de São Paulo'14-16,18,20,22-24,28, seguida por Pernambuco $13,19,21,25,27,29$. Nas pesquisas realizadas em São Paulo, as prevalências de anemia encontradas podem ser consideradas como de moderado a severo nível epidemiológico, segundo proposta da $\mathrm{OMS}^{2}$ para avaliação da prevalência dessa carência. Considerando que esse é o estado mais desenvolvido do Brasil, de acordo com o Índice de Desenvolvimento da Família (IDF), proposto pelo Instituto de Pesquisa Econômica Aplicada (IPEA) ${ }^{32}$, pode-se supor que sua prevalência em estados com menor acesso aos serviços de saúde e com menor desenvolvimento, onde ainda não existem dados suficientes a respeito dessa prevalência, seria ainda mais preocupante.

De acordo com análise temporal das pesquisas realizadas, houve maior enfoque com relação às carências nutricionais a partir da década de 1970 até a década de 1990, coincidindo com o interesse do Brasil em compromissos internacionais para o combate e a prevenção da anemia ${ }^{33,34}$. A partir do ano 2000, observou-se drástica redução no número de estudos sobre anemia, sendo que nenhum dos estudos analisados teve como objetivo principal a descrição da prevalência do problema. Durante a busca bibliográfica foi encontrada quantidade muito maior de dados referentes à anemia em crianças e poucas infor- 
mações sobre mulheres em idade fértil ou gestantes. Pode-se sugerir, ainda, que a prioridade em saúde pública relativa ao tema tem sido direcionada à população infantil.

Provavelmente, isso se deve a três fatores principais: 1) estima-se que não houve redução no número de pesquisas realizadas relativas à anemia, mas o enfoque da maioria dessas pesquisas tem sido em populações outras que não as gestantes (como em especial os bebês entre seis e 24 meses e os pré-escolares); 2) supõe-se que, com o crescente aumento das prevalências de obesidade e de outras doenças crônicas não transmissíveis tem-se dado menor atenção às carências nutricionais como um todo; 3) possivelmente, o período entre os anos 1990 e anterior a 2004 ainda foi um momento em que muitos pesquisadores priorizaram os estudos sobre morbidades que apresentam sintomas clínicos definidos. Nesse período, apenas se inicia o movimento de promoção da saúde e a preocupação com a saúde e nutrição no Brasil. Com a implantação da política de combate à anemia há expectativa de que, cada vez mais estudos sobre o impacto, a eficiência e a efetividade das ações sejam apresentados.

Em uma revisão bibliográfica sobre estudos de prevalência de anemia no Brasil entre 1990 e $2000^{33,34}$, foi comprovado que, apesar de uma primeira conclusão ser a de que o número de estudos realizados caracteriza bem o problema no País, os estudos são restritos a populações específicas, não sendo possível extrapolar esses resultados. Além disso, dos 18 estudos encontrados, apenas três tiveram base populacional em mulheres em idade fértil e apenas um focou gestantes.

\section{O N CLUS Ã O}

Os estudos realizados nos últimos 40 anos referentes à prevalência de anemia na população de gestantes no Brasil são escassos e centralizados em determinadas regiões, especialmente os estados de São Paulo e Pernambuco. Esse fato dificulta estimar com precisão a prevalência dessa carência nesse estado fisiológico no País. Além disso, cada estudo utilizou um método diferente ou próprio, inclusive admitindo outros pontos de corte para a classificação, dificultando a comparação de dados. Chama a atenção, dessa forma, a baixa representatividade dos artigos relativos à anemia na gestação encontrados no Brasil e mesmo em outros países. Os estudos encontrados foram poucos e com amostras pequenas e restritas.

A formulação da política nacional de fortificação de farinhas de trigo e de milho com ferro e ácido fólico, de $2002^{35}$ foi embasada em dados da prevalência de anemia em crianças brasileiras dos anos 1970 a 1990. Observa-se que, com base nas informações de prevalência de anemia ou de deficiência de ferro na população de gestantes, essa política vem em momento oportuno como uma estratégia já utilizada por outros países 2,4,36 para o combate à carência. A partir da implantação dessa política de redução de anemia, estudos contemplando a prevalência de anemia, a associação de variáveis e o impacto da estratégia de fortificação nas populações brasileiras têm sido realizados ${ }^{37-41}$, alguns deles já publicados em revistas científicas brasileiras. Sugere-se que tais trabalhos sejam cada vez mais estimulados, de forma que sejam construídos parâmetros brasileiros de comparação.

\section{COLABORADORES}

M.H. CÔRTES E I.A.L. VASCONCELOS, responsáveis pela pesquisa e pela interpretação de dados. D.C. COITINHO, responsável pela concepção e pela orientação do estudo.

\section{REFERÊ NCIAS}

1. Centers for Diseases Control and Prevention. Recommendations to prevent and control iron deficiency in the united states morbidity and mortality weekly report, 47(3) [e-journal]. Atlanta, 1998 [cited 2008 Jun 24]. Available from: <http:// www.cdc.gov/mmwr/preview/mmwrhtml/ 00051880.htm>.

2. World Health Organization. Iron deficiency anaemia: assessment, prevention and control. A guide for programme managers. Geneva; 2001 
[cited 2008 Jun 24]. Available from: <http://www. who.int/nutrition/publications/en/ida_ assessment_prevention_control.pdf $>$.

3. Barón MA, Solano L, Peña E, Sãnchez A, Real SD. Estado de las reservas de hierro al inicio del embarazo. Invest Clín. 2005; 46(2):121-30.

4. World Health Organization. Assessing the iron status of populations. Report of a Joint World Health Organization/Centers for Disease Control and Prevention Technical Consultation on the Assessment of Iron Status at the Population Level. Geneva, 2004 [cited 2006 May 27]. Available from: <http://whqlibdoc.who.int/publications/2004/ 9241593156_eng.pdf>.

5. Bottoni A, Ciolette A, Schmitz BAS, Campanaro CM, Accioly E, Cuvello LCF. Anemia ferropriva. Rev Paul Pediatr. 1997; 15(3):127-34.

6. World Health Organization. Vitamin and mineral requirements in human nutrition. 2 nd ed. Bangkok: WHO; 1998 [cited 2006 Jun 1]. Available from: <http://whqlibdoc.who.int/publications/ 2004/9241546123.pdf>.

7. Freire WB. La anemia por deficiência de hierro: estratégias de la OPS/OMS para combatirla. Salud Pub Mex. 1998; 40(2):199-205.

8. Vítolo MR. Nutrição da gestação à adolescência. Rio de Janeiro: Reichmann e Affonso Editores; 2003.

9. Allen LH. Advantages and limitations of iron amino acid chelates as iron fortificants. Nutr Rev. 2002; 60(7 Suppl 2):S18-S21.

10. Ribeiro JAC, Soares CB, Janarelli AL. Anemias na gestação. Ginecol Obstetr Atual. 1995; 4(1/2):34.

11. Szarfarc SC. Anemia ferropriva em populações da região sul do estado de São Paulo. Rev Saúde Pública. 1972; 6:125-33.

12. Rodriguez OT. Estudo da Influência de anemia de desnutrição maternas sobre o peso ao nascer [dissertação]. São Paulo: Universidade de São Paulo; 1989.

13. Arruda IKG. Prevalência de anemia em gestantes de baixa renda: algumas variáveis associadas e sua repercussão no recém-nascido [tese]. Recife: Universidade Federal de Pernambuco; 1990.

14. Cook JD, Alvarado J, Gutnisky A, Jamra M, Lambardini J, Layrisse M, et al. Nutritional deficiency and anemia in Latin América: a collaborative study. Blood. 1971; 38(5):591-603.

15. Almeida PAM, Ciari Jr C, Santos JLF, Siqueira AAF. Curva de hemoglobina em um grupo de gestantes normais. Rev Saúde Pública. 1973; 7(3):273-82.

16. Szarfarc SC. Anemia ferropriva em parturientes e recém-nascidos. Rev Saúde Pública. 1978; 8(4): 369-74.
17. Vaz Pinto A, Pinto GP, Formiga Filho JFN, Lara S, Santos F. A anemia da gravidez em Sobradinho, cidade satélite de Brasília, Brasil. Rev Bras Pesq Méd Biol. 1975; 8(5-6):381-5.

18. Roncada MJ, Szarfarc SC. Hipovitaminose A e anemia ferropriva em gestantes de duas comunidades do vale do Ribeira (Estado de São Paulo, Brasil). Rev Saúde Pública. 1975; 9(2):99-106.

19. Salzano AC, Batista Filho M, Flores H, Calado CLA. Prevalência de anemia no ciclo gestacional em dois estados do nordeste brasileiros, Pernambuco e Paraíba. Rev Bras Pesq Méd Biol. 1980; 13(4-6): 211-4.

20. Szarfarc SC, Siqueira AAF, Martins IS, Tanaka ACD' A. Estudo comparativo de indicadores bioquímicos da concentração de ferro, em duas populações de gestantes, com e sem atendimento pré-natal. Rev Saúde Pública. 1982; 16(1):116.

21. Romani SAM, Torres MAA, Batista Filho M, Salzano AC. Anemias em gestantes de duas unidades de saúde da cidade de Recife-PE. Rev Bras Malariol Doenças Trop. 1984; 36:1-10.

22. Szarfarc SC. A anemia nutricional entre gestantes atendidas em centros de saúde do Estado de São Paulo (Brasil). Rev. Saúde Pública. 1985; 19(5): 450-7.

23. Guerra EM, Barretto, OCD, Vaz, AJ, Silveira, MB. Prevalência de anemia em gestantes de primeira consulta em centros de saúdes de área metropolitana, Brasil. Rev Saúde Pública. 1990; 24(5): 380-6.

24. Sinisterra-Rodriguez OT. Anemia nutricional en el embarazo. Rev Hosp Niño (Panamá). 1990; 9(2): 121-4.

25. Nacul LC, Lira PI, Batista Filho M. Anemia em gestantes atendidas no pré-natal do IMIP. Rev IMIP. 1991; 4(2):104-7.

26. Cardoso MA, Ferreira MU, Camargo LMA, Szarfarc SC. Anemia em população de área endêmica de malária, Rondônia (Brasil). Rev Saúde Pública. 1992; 26(3):161-6.

27. Arruda IKG. Deficiência de ferro e folato e anemia em gestantes atendidas no IMIP: magnitude, alguns fatores de risco e repercussão nos seus conceptos [tese]. Recife: Universidade Federal de Pernambuco; 1997.

28. Rondó PH, Thomkins AM. Maternal iron status and intrauterine growth retardation. Trans R Soc Trop Med Hyg. 1999; 93:423-6.

29. Souza Al, Ferreira LOC, Batista Filho M, Dias MRFS. Enteroparasitoses, anemia e estado nutricional em grávidas atendidas em serviço público de saúde. Rev Bras Ginecol Obstet. 2002; 24(4):253-9. 
30. Rocha DS, Netto MP, Priore SE, Lima NMM, Rosado LEFPL, Franceschini SCC. Estado nutricional e anemia ferropriva em gestantes: relação com o peso da criança ao nascer. Rev Nutr. 2005; 18(4):481-9.

31. Szarfarc SC. Densidade do ferro biodisponível em uma dieta habitual no estado de São Paulo. Rev Saúde Pública 1983; 17(4):290-6.

32. Instituto de Pesquisa Econômica Aplicada. Texto para discussão $n^{\circ} 986$. Índice de Desenvolvimento da Família. Rio de Janeiro; 2003 [acesso 2008 jun 24]. Disponível em: <http://www.ipea.gov.br/pub/ td/2003/td_0986.pdf>.

33. Organização Pan-Americana de Saúde. Bibliografia sobre deficiência de micronutrientes no Brasil 1990-2000. Brasília; 2002. v.2: Anemia [acesso 2008 jun 24]. Disponível em: <http://www.opas. org.br/sistema/arquivos/pes_vol2a.pdf>.

34. Organização Pan-Americana de Saúde. Bibliografia sobre deficiência de micronutrientes no Brasil 1990-2000. v.2b: Anemia [acesso 2008 jun 24]. Disponível em: <http://www.opas.org.br/sistema/ arquivos/pes_vol2b.pdf>.

35. Brasil. Agência Nacional de Vigilância Sanitária. Resolução n 344, de 13 de dezembro de 2002. Aprova o regulamento técnico para a fortificação das farinhas de trigo e milho com ferro e ácido fólico [acesso 2003 out 21]. Disponível em: <http:// www.anvisa.gov.br/legis/resol/2002/344_02 rdc.htm>
36. David LJ. Fortificación de harina de trigo en América Latina y región del Caribe [Cartas ao Editor]. Rev Chil Nutr. 2004; 31(3):336-47.

37. Côrtes $\mathrm{MH}$. Impacto da fortificação das farinhas de trigo e de milho com ferro nos níveis de hemoglobina das gestantes atendidas pelo pré-natal do hospital universitário de Brasília/DF [dissertação]. Brasília: Universidade de Brasília; 2006.

38. Vasconcelos IAL. Avaliação do consumo de alimentos sujeitos à fortificação compulsória com ferro das gestantes atendidas no pré-natal do hospital universitário de Brasília, Distrito Federal [dissertação]. Brasília: Universidade de Brasília; 2006.

39. Araciaba A. Impacto da fortificação compulsória das farinhas de trigo e milho com ferro, nos níveis de hemoglobina de crianças de 12 a 24 meses, Sobradinho, Distrito Federal, Brasil [dissertação]. Brasília: Universidade de Brasília; 2005.

40. Assunção MCF, Santos IS, Barros AJD, Gigante DP, Victora CG. Efeito da fortificação de farinhas com ferro sobre anemia em pré-escolares, Pelotas, RS. Rev Saúde Pública. 2007; 41(4):539-48.

41. Vasconcelos IAL, Côrtes MH, Coitinho DC. Alimentos sujeitos à fortificação compulsória com ferro: um estudo com gestantes. Rev Nutr. 2008; 21(2):149-60.

Recebido em: 11/12/2006

Versão final reapresentada em: 6/8/2008

Aprovado em: 18/2/2009 